\title{
An improved sampling rule for mapping geopotential functions of a planet from a near polar orbit
}

\author{
Matthias Weigelt - Nico Sneeuw - E.J.O. Schrama - Pieter N.A.M. Visser
}

Received: date / Accepted: date

\begin{abstract}
One of the limiting factors in the determination of gravity field solutions is the spatial sampling. Especially during phases, when the satellite repeats its own track after a short time, the spatial resolution will be limited. The Nyquist rule-of-thumb for mapping geopotential functions of a planet, also referred to as the Colombo-Nyquist rule-ofthumb, provides a limit for the maximum achievable degree of a spherical harmonic development for repeat orbits. We show in this paper that this rule is too conservative and solutions with better spatial resolutions are possible. A new rule is introduced which limits the maximum achievable order (not degree!) to be smaller than the number of revolutions if the difference between the number of revolutions and the number of nodal days is of odd parity and to be smaller than half the number of revolutions if the difference is of even parity. The dependence on the parity is reflected in the eigenvalue spectrum of the normal matrix and becomes especially important in the presence of noise. The rule is based on applying the Nyquist sampling theorem separately in North-South and East-West direction. This is only possible for satellites in highly inclined orbits like e.g. CHAMP and GRACE. Tables for these two satellite missions are also provided which indicate the passed and (in case of GRACE) expected repeat cycles and possible degradations in the quality of the gravity field solutions.
\end{abstract}

Keywords Spatial sampling - space-borne gravimetry · Nyquist rule-of-thumb $\cdot$ CHAMP $\cdot$ GRACE

\section{Weigelt, N. Sneeuw}

Institute of Geodesy, Universität Stuttgart

Geschwister-Scholl-Str. 24D, 70174 Stuttgart, Germany

E-mail: weigelt@ gis.uni-stuttgart.de

E.J.O. Schrama, P.N.A.M. Visser

Delft Institute of Earth Observation and Space Systems (DEOS)

Delft University of Technology, Kluyverweg 1, 2629 HS,

Delft, The Netherlands

\section{Introduction}

The quality of a satellite derived gravity field solution depends on the spatial and temporal sampling of the signal. Generally, the sampling rate describes the repeat rate of taking observations over a domain. In the case of spatial sampling the domain is the Earth, i.e. it describes the spatial distribution of the observations on the Earth. In this paper the temporal sampling is referred to as the temporal resolution of global gravity field solutions (and not to the sampling rate of the observation along the orbit), i.e. a gravity field solution is derived from observations taken in a period of time, e.g. one week or one month, and is then considered as one sample.

The Nyquist sampling theorem states that the maximum reconstructible frequency is half the sampling rate $f_{s}$. Signals with frequencies higher than $f_{s} / 2$ are undersampled and cause aliasing. Temporal aliasing thus refers here to the undersampling of the time-variable gravity changes induced by mass transports, e.g. Ilk et al. (2005). It is considered as one of the major limitations for the Gravity Recovery and Climate Experiment (GRACE) (Tapley et al., 2004; Han et al., 2004). A more detailed discussion about this type of aliasing is given in Visser et al. (2010). Here, we restrict ourselves to the spatial sampling.

The maximum spatial resolution depends on a combination of the sensitivity of the measurement system and the orbit configuration. Colombo (1984) formulated a Nyquisttype of rule where he assumed that for a recovery of the gravity field with the maximum degree $L$ at least $2 L$ nonoverlapping revolutions are necessary. Consequently, the maximum resolvable degree for a particular orbit configuration is given as

$L<\frac{\beta}{2}$, 
where $\beta$ denotes the number of orbits in a so-called repeat cycle. It is implicitly assumed that the $2 L$ revolutions are equally spread at the equator. A repeat cycle is achieved if the satellite completes $\beta$ revolutions in an integer number of nodal days, which is the number of revolutions of the orbital plane around the $\mathrm{z}$-axis of the Earth-fixed coordinate system. It is generally denoted as the ratio $\beta / \alpha$ which is an integer ratio and where common prime factors vanish. In Schrama (1989) sampling properties of a satellite in a $\beta / \alpha=-43 / 3$ repeating ground track orbit are worked out in more detail, i.e. an analytical orbit perturbation theory is compared against a numerically generated result. The negative sign denotes a retrograde orbit. The interpretation of the ratio is that within a repeat cycle lasting $\beta=43$ orbits, the orbital plane rotated $\alpha=3$ times. Schrama (1991) discusses in more detail that by ignoring the rule given in equation (1) specific frequencies cannot be recovered anymore since in a lumped coefficient approach certain combinations of the wavenumber $k$ and the order $m$ map to the same frequency and are thus not separable anymore. These analytical approaches are often amended by numerical investigations, e.g. Yamamoto et al. (2005); Bezděk et al. (2009, 2010)

Equation (1) is generally accepted as the Nyquist ruleof-thumb for mapping geopotential functions of a planet, often also referred to as the Colombo-Nyquist rule-of-thumb, which connects the spatial resolution with the sampling. It found wide-spread application in the orbit design and recovery of the gravity field, e.g. Johannessen and AguirreMartinez (1999) or Bender et al. (2008). Since the motion of the satellite governs the ratio, it can be determined using the rate of change of the Keplerian elements, in particular the true anomaly $v$, the argument of perigee $\omega$ and the right ascension of the ascending node $\Omega$.

$\frac{\beta}{\alpha}=\frac{\dot{\omega}+\dot{v}}{\dot{\Omega}-\omega_{\mathrm{E}}}=-\frac{\dot{u}}{\dot{\Lambda}}=\frac{T_{\Lambda}}{T_{u}}$,

where $\omega_{\mathrm{E}}$ is the angular velocity of the Earth rotation. The sum of $v$ and $\omega$ forms the argument of latitude $u$; the sum of the denominator can be seen as the rate of change of the longitude of the ascending node in the Earth fixed frame $\Lambda$. The ratio is also connected to the revolution time of the satellite $T_{u}$ and of the nodal day $T_{\Lambda}$.

Repeat cycles are of special interest for gravity field missions. On the one hand, they can be used to recover specific orders of coefficients with high quality (Wagner and Klosko, 1977; Klokočník et al., 1990). On the other hand, they can cause a degradation of the overall performance due to an insufficient spatial sampling. The influence of the ground track on the quality of the solution attracted first attention for the low-low satellite-to-satellite tracking mission GRACE and has been investigated by Yamamoto et al. (2005) using simulated data. Wagner et al. (2006) compared the severe loss of accuracy of monthly solutions to degree and order 120 of published GRACE solutions during the 61/4-resonance orbit in September 2004 to theoretical error estimates from linear perturbation theory. They concluded that the ideal maximum degree is $L=30$ which is in agreement with the rule-ofthumb. Klokočník et al. (2008) extended the investigations of Wagner et al. (2006) to the cases of CHAMP and GOCE and predicted future periods of degraded performance of GRACE.

Recently, Weigelt et al. (2009) noted a discrepancy between the maximum recoverable degree for CHAMP as the satellite passed on several occasions through the 31/2 repeat cycle. The rule-of-thumb predicts a maximum recoverable degree of 15 but the solutions remained valid approximately till degree and order of 35. Visser et al. (2012) showed by means of numerical calculations that the rule-of-thumb is too stringent for geodetic type of satellite missions like CHAMP, GRACE and GOCE. Instead, he formulated the condition

$L<\beta$

for one-dimensional quantities like satellite altimetric observations, potential values along the orbit or a single gradiometer component. For other types of observations like accelerations and the full tensor components, solutions higher than degree and order $L=\beta$ are possible.

Here, we consider again the case of a one dimensional type of observation but instead of using geoid values along a satellite ground path (similar to altimeter observations) we use potential values along the orbit, i.e. the downward continuation will influence the quality of the solution as well. We restrict ourselves to orbits with inclinations close to $90^{\circ}$ in order to separate the influence of polar gaps from the properties and influences of repeat configurations. For the polar gap problem we refer to the literature, e.g. Sneeuw and van Gelderen (1997) or Albertella et al. (1999).

The sampling is generally defined by the geometry of the satellite ground tracks and a discussion about the fundamental geometry of those can be found in e.g. Kim (1997). For satellite missions in near polar orbits, the sampling can be investigated independently in longitude and latitude direction. In flight direction $(\approx$ latitude direction $)$, the sampling is governed by the measurement rate of the observations, e.g. GPS positions or the K-band ranging system, and normally dense and stable over time. The sampling in longitude direction is laid out by the orbit on the rotating Earth and can be described by the number of equator crossings. In such a configuration one easily undersamples the Earth as coarse spacings between ascending and descending arcs occur especially during repeat cycles with low integer values for $\beta$ and $\alpha$. Using Nyquist-type criteria in both directions, it will be shown that a restriction on the maximum spatial resolution due to the orbit configuration exists mostly on the maximum resolvable order $M$ rather than on the max- 
imum resolvable degree $L$ as proposed in Colombo (1984) and Visser et al. (2012).

Further, a dependency on the parity of $(\beta-\alpha)$ exists, since the result of even parity degrade due to a poorer conditioning of the normal matrix. This has already been recognized in Visser et al. (2012) but there the focus laid on the solvability of the equation system and the interpretation of the formal errors. Here the quality of the solution is considered additionally and it will be shown that a valid solution is achievable in this case but with a reduced quality. For highly precise application like GRACE, this cannot be neglected and a new type of criterion is introduced which connects the maximum resolvable order $M$ to the number of so-called unique equator crossings.

Section 2 starts with a theoretical description of the orbit geometry and its connection to the sampling which eventually leads to a new sampling criterion. The dependency of the relative location of equator crossings of ascending and descending arcs on the parity $(\beta-\alpha)$ during a repeat cycle is introduced in section 2.1. Using the time series representation for the spherical harmonics, a Nyquist-type criterion can be applied which considers also the aforementioned dependency on the parity (section 2.2). The criterion is verified by numerical noise-free simulation in sections 3.1 and 3.2. In section 3.3, noise is added to the simulation and the degradation in the case of even parity is deduced. We conclude with a discussion on the implications for the CHAMP and GRACE mission in section 4.

\section{Theoretical Aspects}

Since satellites like CHAMP and GRACE are in a near polar orbit, the sampling of the Earth can in good approximation be split into two components. Measurements along ascending or descending arcs sample the gravity field in NorthSouth (latitude) direction, whereas the sampling in East-West (longitude) direction is governed by the distribution of the arcs. A measure of which is the separation between equator crossings and their distribution depends on the orbit configuration. Normally, the measurement rate, i.e. the sampling in latitude direction, is much higher than the number of equator crossings. Especially during repeat cycles with low relative prime numbers $\beta$ and $\alpha$, the sampling in longitude direction becomes sparse. An example for CHAMP taken from Weigelt et al. (2009) is shown in Fig. 1. The solid grey line represents the signal, e.g. the disturbing potential, along a particular latitude ( $\phi=80^{\circ}$ on the top and the equator on the bottom). The observations of CHAMP are marked by black triangles. The left column shows the case of January 2004 when the satellite sampled the Earth with an evenly spaced data distribution at all latitudes. In June 2003 the satellite was near the $31 / 2$ repeat mode and the corresponding sampling is depicted in the right column of Fig. 1. The data separation at high latitudes changes only slightly whereas the observations at the equator become clustered and sparsely distributed. Several publications showed that this causes a severe quality degradation of the monthly gravity field solutions, e.g. Sneeuw et al. (2003, 2005); Weigelt et al. (2009). As a consequence, the sampling at the equator and in the longitude direction is of primary concern in the following.

\subsection{Geometry of the equator crossings}

Although ascending and descending arcs cross the equator each $\beta$ times, the sampling in the longitude direction at the equator is obviously halved if crossings of ascending and descending arcs coincide. The condition for this case can be connected to the parity of $(\beta-\alpha)$.

Consider a circular orbit and an arbitrarily located equator crossing of an ascending arc at longitude $\lambda_{0}^{\mathrm{a}}$. The equator crossing of the $p$-th ascending arc can then be found by:

$\lambda_{p}^{\mathrm{a}}=\lambda_{0}^{\mathrm{a}}+\frac{2 \pi}{\beta} p$, with $p=0 \ldots \beta-1$,

since the equator crossings of all ascending arcs will be equally spread. In this description, the value of $p$ is not connected to the temporal occurrence of the equator crossing but to the numbering from West to East, i.e. the numbering is only possible after one repeat cycle has been finished.

Starting from $\lambda_{0}^{\mathrm{a}}$, the location of the first (in the timely sense) equator crossing of a descending arc is shifted by 180 degrees minus the angle passed by the Earth during a half revolution of the satellite.

$\lambda_{0}^{\mathrm{d}}=\lambda_{0}^{\mathrm{a}}+\pi-\frac{1}{2} \omega_{\mathrm{E}} T_{u}$.

The minus sign is due to the counter-clockwise rotation of the Earth (as observed at the North pole). Since the rotation

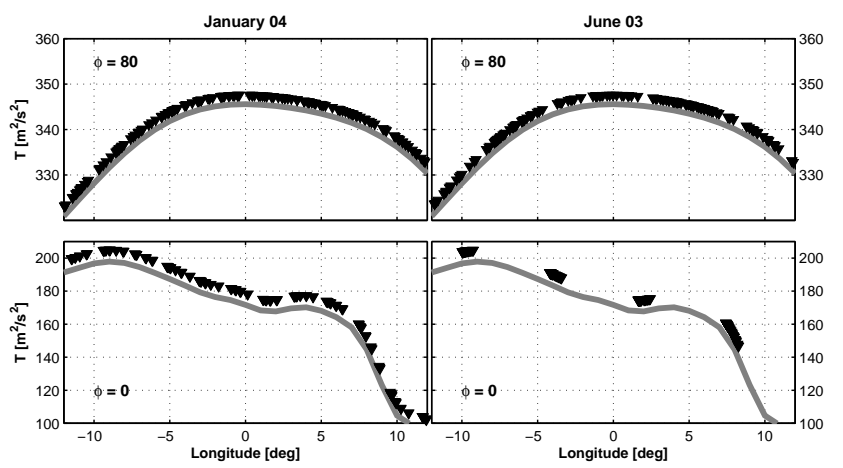

Fig. 1 Sampling (black triangles) of the disturbing potential (solid grey line) for a $25^{\circ}$ degree section around the Greenwich meridian: left column for January 2004 and right column for June 2003; top row for a high-latitude parallel $\left(\phi=80^{\circ}\right)$, bottom row for the equator $\left(\phi=0^{\circ}\right)$; from Weigelt et al. (2009) 
rate $\omega_{\mathrm{E}}$ describes one revolution of the Earth in one nodal day $T_{\Lambda}$, it can be replaced by the ratio $\frac{2 \pi}{T_{\Lambda}}$. Equation (2) connects the ratio $\frac{T_{u}}{T_{\Lambda}}$ to the inverse of the repeat ratio $\beta / \alpha$, i.e.

$\frac{1}{2} \omega_{\mathrm{E}} T_{u}=\pi \frac{\alpha}{\beta}$

The equator crossings of the descending arc is then given by:

$\lambda_{0}^{\mathrm{d}}=\lambda_{0}^{\mathrm{a}}+\pi-\pi \frac{\alpha}{\beta}$

The condition that this particular descending arc coincides with any of the ascending arcs is then given as:

$\lambda_{p}^{\mathrm{a}}-\lambda_{0}^{\mathrm{d}}=0$

If this condition is fulfilled for one descending arc it is also fulfilled for all other. Thus, it is sufficient to proof this for this single case. The more general case is given in appendix A. Combining equation (4) and (7) yields

$\lambda_{0}^{\mathrm{a}}+\frac{2 \pi}{\beta} p-\lambda_{0}^{\mathrm{a}}-\pi+\pi \frac{\alpha}{\beta}=0$

$\Leftrightarrow \quad \frac{2 p}{\beta}-1+\frac{\alpha}{\beta} \quad=0$

$\Leftrightarrow 2 p-\beta+\alpha \quad=0$

$\Leftrightarrow \quad \quad \quad=\frac{1}{2}(\beta-\alpha)$

Since all quantities are integer values, equation (9) has only a solution in case of even parity of $(\beta-\alpha)$. Geometrically it means that in this case the equator crossings of ascending and descending arcs coincide whereas in case of $(\beta-\alpha)$ being odd the equator crossings of the descending arcs are located inbetween the ascending arcs.

Fig. 2 shows exemplarily the dependence of the spatial sampling on the parity. In the left panel, the ground track pattern is shown for the repeat ratio $46 / 3$ (odd parity) and in

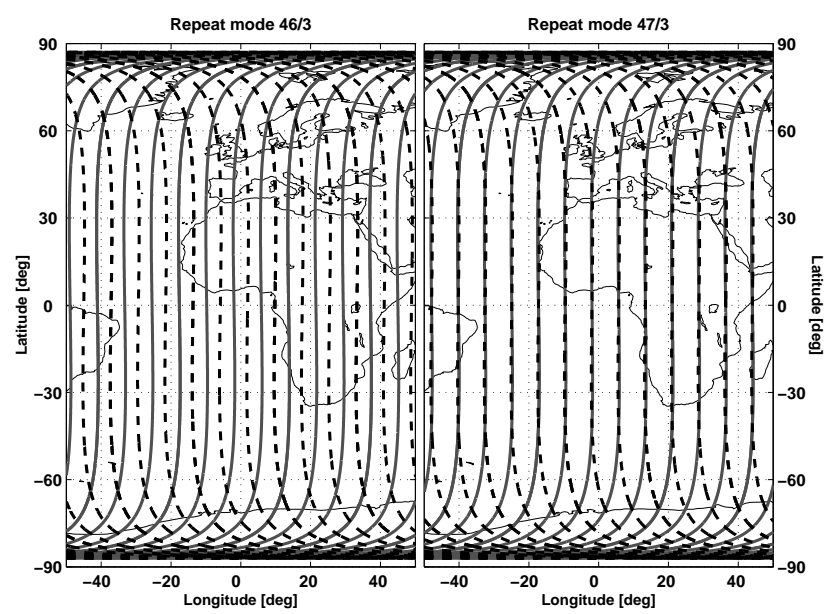

Fig. 2 Equator crossings of a satellite with odd parity (left) and with even parity (right) the right panel for the repeat cycle $47 / 3$ (even parity). Clearly, the case of odd parity results in a denser spatial coverage. The difference is caused by the distribution of the equator crossings and not by the number of equator crossings; it is nearly the same in both cases: 94 for the even case and 92 for the odd case. As a consequence it is more appropriate to speak of the number of unique equator crossings, denoted as $\chi$ in the following, where crossings at the same longitude are only counted once.

$\chi= \begin{cases}2 \beta, & \text { for } \beta-\alpha \text { odd } \\ \beta, & \text { for } \beta-\alpha \text { even. }\end{cases}$

Practically, equator crossings of ascending and descending arcs will seldom be located exactly at the same longitude due to disturbances of the orbit. Equation (8) can only be fulfilled approximately and unique equator crossing need then to be defined by a threshold, e.g.

$\lim _{\varepsilon \rightarrow 0}\left|\lambda_{p}^{\mathrm{a}}-\lambda_{0}^{\mathrm{d}}\right|<\varepsilon$

The choice of $\varepsilon$ is not trivial. A starting point can be based on the anticipated maximum degree $L$ of the mission or the maximum recoverable degree during a period of good ground track coverage, i.e. the spatial sampling can be excluded as limiting factor.

In case of even parity, ascending and descending arcs coincide resulting in a sparser sampling of the equator in East-West direction. In this sense and for the description of the sampling, it is correct to say that the sampling doubled in the odd case. Besides, the convergence of the orbit towards the poles results in a increasingly denser sampling. It is reflected in the nearly constant sampling at high latitudes, as shown in Fig. 1, but the sampling at the equator will be the limiting factor for global gravity field recovery, as mentioned before.

\subsection{Time series criterion}

In an ideal case, the sampling can be connected to a maximum resolvable frequency. For a time series, this is defined by the Nyquist rule. For the sampling of the sphere, the ruleof-thumb needs refinement as shown by Weigelt et al. (2009) and Visser et al. (2012). The first choice at hand is then to try to use the Nyquist criterion itself. For this, the spatial representation of the spherical harmonic development needs to be expressed as a time series. A detailed description of which can be found in Schrama $(1990,1991)$ and Sneeuw (2000). The result is a time series representation of the potential in complex notation:

$V(t)=\frac{G M}{R} \sum_{l=0}^{L} \sum_{m=-l}^{l} \sum_{k=-l}^{l}\left(\frac{R}{r}\right)^{l+1} \bar{K}_{l m} \bar{F}_{l m k}(I) e^{i \psi_{m k q}(t)}$, 
where $G M$ is the gravitational constant times the Earth's mass, $R$ the Earth radius, $\left(\frac{R}{r}\right)^{l+1}$ the upward continuation term, $\bar{K}_{l m}$ the complex-valued spherical harmonic (SH) coefficients, $\bar{F}_{l m k}$ the complex-valued inclination function, $I$ the inclination and $i$ the imaginary number. The summation indices are the degree $l$, the order $m$, the wave number $k$ and the summation index $q$. The maximum degree of the development is $L$. In the following, we assume a negligible eccentricity.

Assuming near-circular orbits (terms with $q \neq 0$ can be neglected) and introducing the linearizing

$\psi_{m k}(t)=\psi_{m k}^{0}+\dot{\psi}_{m k} \cdot t$

the angular variable $\psi$ represents the spectral lines of the lumped coefficient spectrum. (Sneeuw, 2000, §5.3):

$\dot{\psi}_{m k}=k \dot{u}+m \dot{\Lambda}$, with $-L \leq m, k \leq L$.

The initial state of the angular variable $\psi_{m k}^{0}$ is assumed to be zero. In order to avoid spatial aliasing, the separation condition of two frequencies $\dot{\psi}_{m_{1}, k_{1}} \neq \dot{\psi}_{m_{2}, k_{2}}$ must be met. A transformation using equation (2) yields:

$\frac{m_{1}+m_{2}}{k_{1}+k_{2}}=-\frac{\dot{u}}{\dot{\Lambda}} \neq \frac{\beta}{\alpha}$

Colombo (1984) concluded from this condition that for a given maximum degree $L$ at least $\beta$ revolutions are necessary in order to avoid aliasing. Sneeuw (2000) showed that this is fulfilled if

$\beta>2 M$

which he states to be equivalent to the Nyquist rule-of-thumb used by Colombo (1984) in the conventional triangular SH domain. Nonetheless, equations (15) and (16) imply that the limited spatial sampling does not restrict the maximum degree $L$ directly, since $l$ does not appear in the separation condition explicitly. Instead, the limitation is on the maximum order $M$.

\subsection{Spatial criterion}

In the conventional representation of the spherical harmonic development in the spatial domain the sine and cosine term depend solely on the order $m$ and the longitude $\lambda$, cf. Heiskanen and Moritz (1967). The maximum order $M$ is thus directly connected to the sampling in longitude direction. As shown in section 2.1, this sampling is defined by the number of unique equator crossings $\chi$. Equation (16) should thus read:

$\chi>2 M$.
Applying the Nyquist criterion, this means practically that the maximum resolvable order is given by

$M<\beta \quad$ for $\beta-\alpha$ odd

$M<\frac{\beta}{2} \quad$ for $\beta-\alpha$ even,

since $\chi$ depends on the parity of $(\beta-\alpha)$. Equation (18) guarantees that no aliasing due to an insufficient spatial sampling will occur as long as there is no signal beyond the maximum order $M$. It is emphasized that the stated maximum criteria for the order consider limitations due to the orbit configuration only. Aliasing can still occur due to an insufficient temporal aliasing. It is also an open question how to optimally avoid signal aliasing from outside the bandlimitation given by equation (18). One possibility was explored by Weigelt et al. (2009) by reducing this part of the signal using a priori information. Besides, the signal-to-noise ratio and the quality of background models are other limiting factors for the maximum resolvable spatial resolution but are not considered here. Both questions are beyond the scope of this paper.

A small supplement to the restriction of the maximum degree $L$ needs to be indicated. Equation (16) indeed places no restriction on $L$ but for the particular configurations of the geodetic satellites in near polar orbits the Nyquist criterion can also (independently) be applied in latitude direction. The maximum degree $L$ consequently depends on the measurement rate along the orbit which normally result in less stringent conditions than equation (18). For example, in case of CHAMP with a 30 s measurement rate the maximum degree is $\approx 95$ and for GRACE and its $5 \mathrm{~s}$ measurement rate the maximum degree is $\approx 570$. Both are well beyond the maximum recoverable degree of a (monthly) gravity field solution of these satellites. Moreover and more importantly, they generally remain constant.

\section{Simulation study}

Based on theoretical descriptions, equation (18) yields a new criterion for the maximum order $M$ which needs to be verified by simulations. Since here the primary concern is the sampling of a one-dimensional measurement quantity, it is sufficient to place a CHAMP-type satellite in a Kepleriantype, polar and circular orbit. Assuming the Keplerian case for the orbit dynamics guarantees the exact repeat mode. In reality, the satellite orbit drifts through repeat cycles due to atmospheric drag and other disturbing forces which results already in an improved sampling and better results. Thus this type of simulation represents the worst case scenario.

The orbital elements of the simulation are given in table 1. The semi-major axis $a$ depends on the repeat cycle and is 
calculated by:

$a=\sqrt[3]{\frac{G M}{\omega_{\mathrm{E}}^{2}} \cdot\left(\frac{\alpha}{\beta}\right)^{2}}$,

where $\omega_{\mathrm{E}}$ is again the revolution of the Earth in one nodal day.

Along this orbit and with a stepsize of 30 s, potential values are calculated by a spherical harmonic synthesis using the gravity model EGM08 (Pavlis et al., 2008) and introduced as observations for a subsequent spherical harmonic analysis. In the application to real satellite data, such observations could be derived using the energy integral approach (Jekeli, 1999). Otherwise, different functionals of the gravity field need to be considered (Visser et al., 2012). The results are then derived using a brute-force spherical harmonic analysis on the sphere.

The maximum degree $L$ and order $M$ depends on the simulation setup and is explicitly stated in the following. Primarily, two cases are distinguished. The calculation is either done with the full field $(L=M)$ or with a field which is bandlimited in the order direction $(M<L)$. They are evaluated using the difference between input and solved-for spherical harmonic coefficients and by considering the conditioning of the normal matrix. Condition numbers are calculated as the ratio between maximum and minimum eigenvalue for all test cases with and without band-limitation and are listed in appendix B.

\subsection{Odd parity}

In the first test scenario, the data is assumed to be noise-free. For the case of odd parity, the example of $\beta=46$ and $\alpha=3$ is chosen, i.e. the satellite is repeating its own track after 3 days and 46 revolutions. Using equation (19), the corresponding orbit height is $h=a-R \approx 465.9 \mathrm{~km}$. The maximum order for the bandlimited case is according to equation (18b) $M=45$ since the number of unique equator crossings is $\chi=92$. For comparison, the rule-of-thumb predicts for this case a maximum resolvable degree of $L=23$. The results for different simulation scenarios are presented in terms of the difference spectrum of the real (not complex) spherical harmonic coefficients between input and estimated coefficients and are shown in Fig. 3.

For the chosen CHAMP-type observation, typically a maximum degree of $L=90$ can be achieved in reality and under ideal sampling conditions. The top panel shows the attempt to recover the gravity field till degree and order 90

Table 1 Orbital elements of the simulated satellite orbit at $t=0$

\begin{tabular}{ll|ll} 
eccentricity & $e=0$ & RA of the ascending node & $\Omega=0^{\circ}$ \\
inclination & $I=90^{\circ}$ & $\begin{array}{l}\text { argument of latitude } \\
u=90^{\circ}\end{array}$ \\
\hline
\end{tabular}
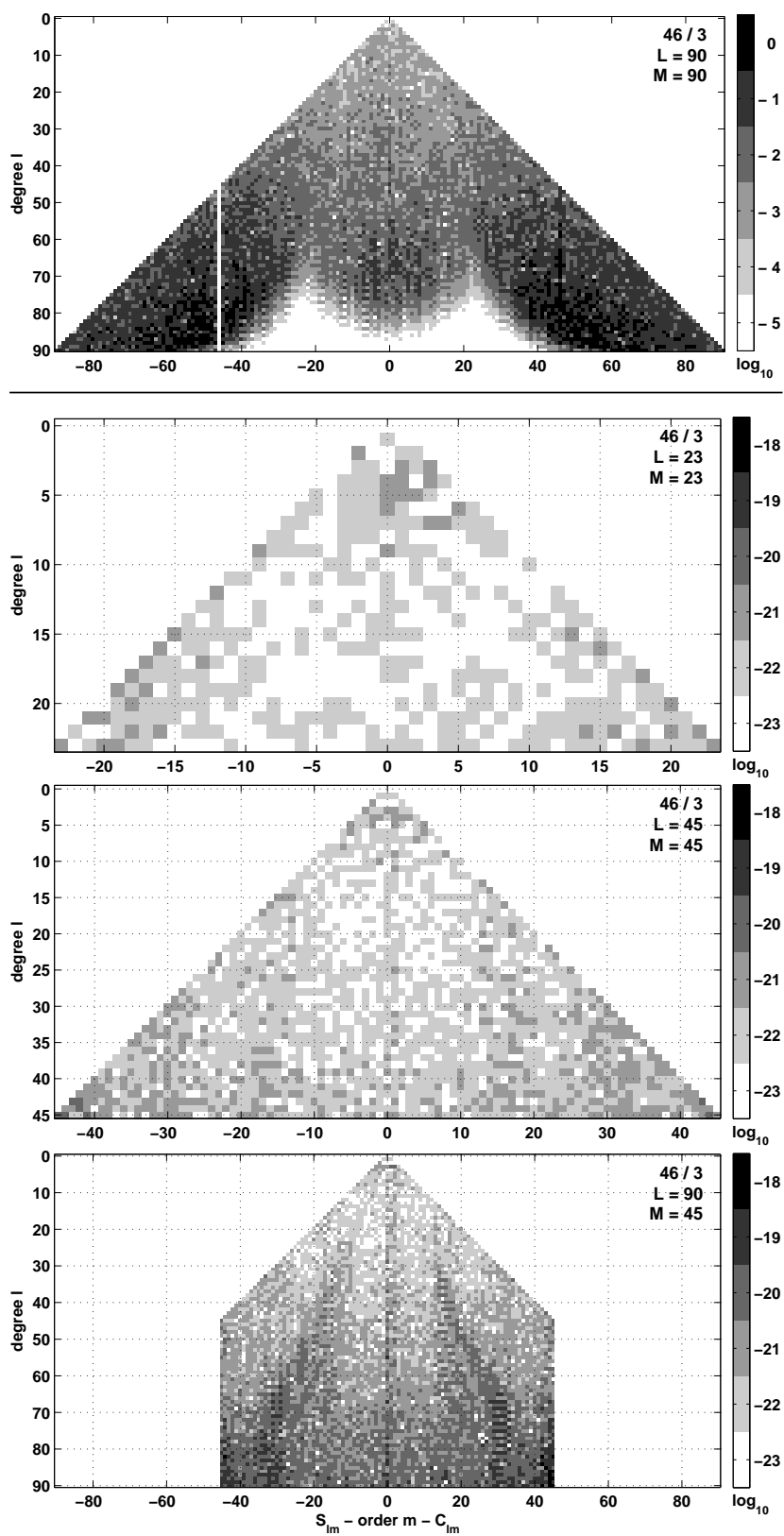

Fig. 3 Spherical harmonic spectrum for $\beta=46$ and $\alpha=3$ : full solution with $L=M=45$ in the top panel, rank deficient solution for $L=M=90$ in the middle panel, order-limited solution with $L=90$ and $M=45$ in the bottom panel. The maximum degree and order is for each case identical for the simulation input and the recovery. Note also the different ranges of the colorbar in the three cases.

but here the sampling conditions are poor as the $46 / 3$ repeat orbit is used. Considering the range of the colorbar, the solution is obviously highly degraded. The condition number for this solution is $10^{23}$, i.e. the solution is numerically rank deficient. The solution has been derived using the MoorePenrose pseudo-inverse for visualization purposes. The attempted recovery is also well beyond the limitations given either by the rule-of-thumb, equation (18) or the ones given in Visser et al. (2012). As an aside, order 46 of the sine co- 
efficients can be recovered comparatively well. It is linked to the orbital frequency and the choice of the right ascension of the ascending node $(\Omega=0)$ and the initial angle for the Greenwich Apparent Siderial Time $(\Theta=0)$ at time $t=0$. Due to this choice, the orbit samples the highs and lows of the sine coefficients of order 46 perfectly, whereas only the roots of the cosine coefficients are observed. This phenomenon is specific for this simulation here and cannot be generalized.

The rule of thumb predicts a maximum degree and order of $L=23$. The second panel in Fig. 3 shows the difference spectrum between input and estimated coefficients for a full field solution $L=M=23$. The differences are on the level of $10^{-18}$ to $10^{-23}$ and only limited by the numerical accuracy of the computation. The sampling in longitude and latitude direction is thus sufficient to fully recover such a field. However, the difference for a simulation with a full field till degree and order 45 shown in the third panel from the top is also on the same level of accuracy, i.e. for the odd case the rule of thumb is to pessimistic as a solution till $L<\beta$ is possible without loss of accuracy. Visser et al. (2012) showed already that the prediction of the rule-of-thumb is too pessimistic which is also verified by this example.

Finally, it needs to be tested that the limitation is on the maximum order $M$ as predicted by equation (18) and in contrast to the conventional interpretation of the rule-of-thumb not on the maximum degree $L$. Therefore, the input field is modified and limited in the order direction to $M=45$ but calculated with a maximum degree of $L=90$. The spectrum of the input signal resembles then a house-like shape. The bottom panel of Fig. 3 shows the difference between the recovered field and the input for this order-limited solution. The difference is again in the range of $10^{-18}$ to $10^{-23}$ and the solution shows the typical degradation with increasing degree. The higher number of unknown parameters result in a slightly poorer conditioning of the normal matrix (cf. table 4). It shows that one key element in the solution is indeed the conditioning of the normal matrix which is generally also influenced by other effects and not only by the orbital configuration. Nevertheless, it can be concluded that also for the order-limited case the solution is only limited by the numerical accuracy of the computation.

Fig. 4 gives a deeper insight into the conditioning of the normal matrix for a range of solutions, either fully developed to a maximum degree and $\operatorname{order} L=M$ or band-limited in the order direction at $M=45$. In case of the full solution $(L=M)$, the conditioning shows a discontinuity at degree 45 which exceeds the floating point accuracy of the computer ( $\sim 16$ digits). The inversion becomes impossible since the normal matrix is numerically rank deficient as already discussed earlier. For the band-limited solutions denoted by $M=45$ no such degradation is observed at order 45 . The simulation confirms consequently the theoretical prediction

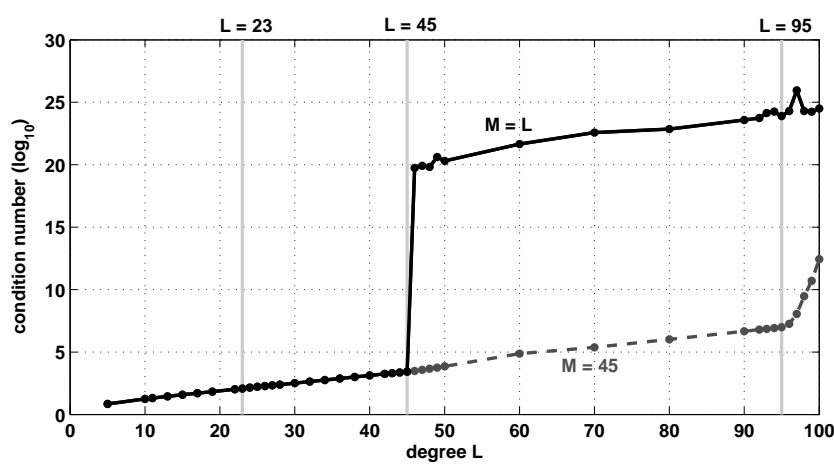

Fig. 4 Condition number of the normal matrices for $\beta=46$ and $\alpha=3$ with full order $(L=M)$ and with order-limitation at $M=45$ : the values on the $\mathrm{x}$-axis indicate the maximum degree $L$ of the $\mathrm{SH}$ - development

of equation (18a) and the solution is primarily limited in the order direction by the sampling in the longitude direction. Near degree 95, the bandlimited solutions also start to degrade as the 30 s-sampling in the latitude direction is insufficient for a higher spatial resolution.

\subsection{Even parity}

For the case of even parity, the example of $\beta=47$ and $\alpha=3$ is chosen due to its close proximity to the previous odd parity case. Although the values for $\alpha$ and $\beta$ are near to the previous case, the orbit configuration changes significantly. Using equation (19), the corresponding orbit height is $h \approx 368.5 \mathrm{~km}$, which is $\approx 100 \mathrm{~km}$ lower than in the previous example. The maximum order for the bandlimited case is $M=23$ according to equation (18b) since the number of unique equator crossings is $\chi=47$.

Fig. 5 shows the difference between reference and estimated model for several cases again. In the top panel, the attempt has been made to recover the gravity field to a maximum degree and order $L=M=90$. Analogously to the odd simulation scenario, the normal matrix is poorly conditioned and a successful recovery is not possible. In the second panel, the maximum degree and order is restricted to $L=M=23$ which is in the agreement with the rule-ofthumb and also fulfills the condition (18b). The solution is only limited by the numerical accuracy of the computation and the sampling is sufficient to fully recover the input field. In the third panel, the input field is extended to degree and order $L=M=46$. This case explicitly tests the difference between equation (18a) for odd parity and equation (18b) for even parity. Obviously the solution slightly degrades compared to the top panel but the degradation seems small. The solution still agrees on a level of $10^{-18}$ at worst. Based on this test no separation as done in equation (18) seems necessary. There is a small systematic effect noticeable but the solution remains perfectly valid and seems primarily only limited by the computationally accuracy. The findings are 

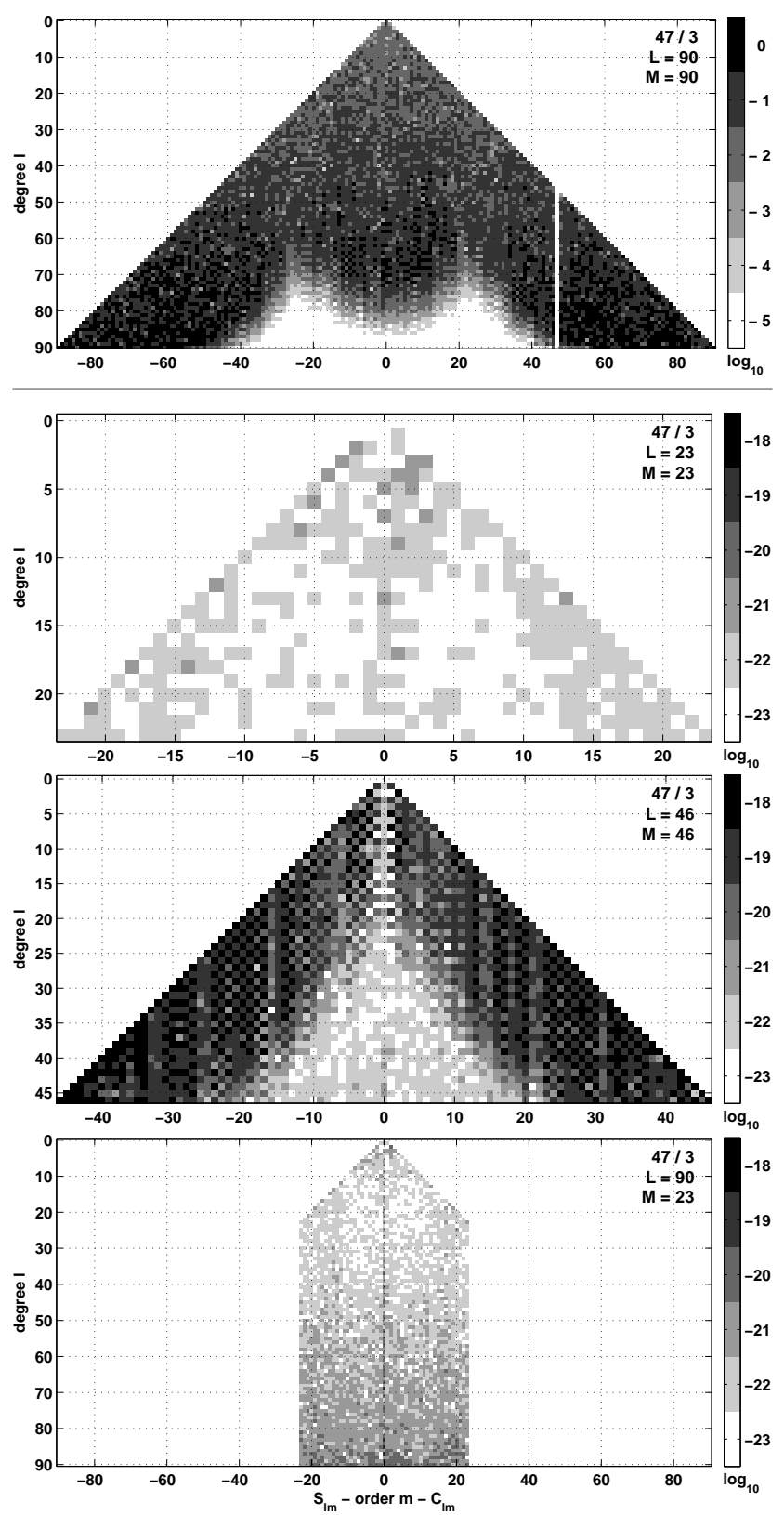

Fig. 5 Spherical harmonic spectrum for $\beta=47$ and $\alpha=3$ : full solution with $L=M=23$ in the top panel, poorer conditioned solution for $L=M=46$ in the middle panel and order-limited solution with $L=90$ and $M=23$ in the bottom panel

thus in agreement with Visser et al. (2012) and equation (3) but we will come back to this point in section 3.3. The bottom panel of Fig. 5 shows the band-limited case. The input field has been limited in the order to $M=23$ but with $L=90$. It can be fully recovered with the same level of accuracy as in the top panel.

The conditioning of the normal matrix, shown in Fig. 6 for the full solution $(L=M)$, a solution band-limited at $M=46$ and one band-limited at $M=23$, indicates the small aforementioned degradation by showing a first discontinuity

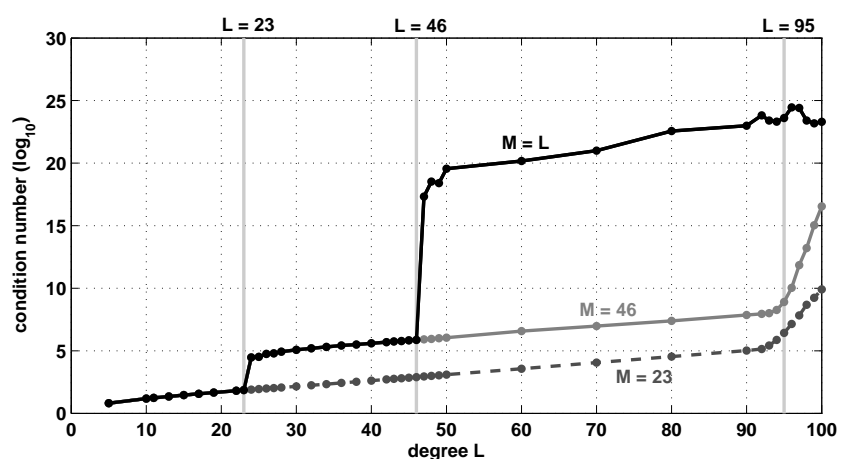

Fig. 6 Condition number of the normal matrices for $\beta=47$ and $\alpha=3$ with full order $(L=M)$ and with order-limitation at $M=46$ and $M=$ 23: the values on the horizontal axis indicate the maximum degree $L$ of the SH - development

at degree 24 for the full solution as well as the one limited at $M=46$. However, the jump is just 2-3 order of magnitudes. The degradation in case of $L=M=46$, visible in the middle panel of Fig. 5, is obviously caused by the poorer conditioning of the normal matrix but the inversion is stable and the solution remains valid. The second discontinuity at degree 46 in case of the full solution $(L=M)$ exceeds the floating point accuracy of the computer and an inversion of the normal matrix is not possible anymore. For the case of the band-limitation at order $M=23$, the condition number shows no discontinuity. Near degree 95 , both band-limited solutions start to degrade as the 30 s-sampling along the orbit is again insufficient for a better spatial resolution.

This confirms the theoretical findings that a Nyquisttype criterion can indeed be used to define restrictions on a maximum resolvable degree and order, respectively, caused by a limited sampling in latitude or longitude direction. So far, the separation for even and odd cases as in equation (18) seems unnecessary. The numerical test showed on the one hand the dependency on the parity but on the other hand that the degradation is small and possibly negligible. The primary effect is the slight deterioration of the condition number by two to three orders of magnitude. As the conditioning of a matrix is especially important in the presence of noise, this case is investigated in the following.

\subsection{Noise impact on the recovery quality}

For the sake of simplicity, white noise is used which is not favoring any specific frequency. Consequently, noise energy is also located beyond the limiting order $M$ and will be aliased. The introduced noise has a standard deviation of $\sigma=1 \mathrm{~m}^{2} / \mathrm{s}^{2}$ which is added to the observations. This level is slightly optimistic compared to in-orbit standard deviations derived from real CHAMP observations $\left(\sigma \approx 1.3 \mathrm{~m}^{2} / \mathrm{s}^{2}\right)$.

For the simulation, the $46 / 3$ and $47 / 3$ orbit configurations are used again. Fig. 7 shows results in terms of difference 
degree RMS with respect to the input field EGM08. Simulations with odd parity $(\beta=46, \alpha=3)$ are denoted in black and with even parity $(\beta=47, \alpha=3)$ in gray. For the case of a full input field with $L=M=23$, the sampling fulfills for both parity cases the condition of equation (18) and results are on the same level of accuracy between $10^{-11}$ and $10^{-12}$.

If the input field is extended to degree and order 45 and 46 , respectively, the odd parity orbit configuration $(\beta=46)$ still fulfills condition (18a) but for the even parity orbit configuration $(\beta=47)$ condition (18b) is not fulfilled. This is the case of the small degradation visible in Fig. 5 and 6 of section 3.2. If the degradation of the conditioning of the normal matrix would be negligible, both cases are expected to be on a similar RMS-level, but the solution of even parity is approximately one order of magnitude worse than for the one of odd parity. The solution of the even parity orbit configuration remains valid though as no strong oscillations or other phenomena are visible in the degree RMS. The difference curve does also not intersect the signal curve. This is in agreement with the findings of Visser et al. (2012), i.e. it is possible to derive a solution till degree and order 46 in case of the insufficient ground track but the solution will be of poorer quality. The band-limited solution for the even orbit configuration with degree $L=90$ and order $M=23$ on the other hand has a level of accuracy comparable to the case of the orbit with odd parity and $L=M=45$, thus verifying equation (18). Consequently, the degradation in the condition number for the even cases cannot be neglected in highly precise applications like GRACE.

Last but not least, the band-limited solution for the odd orbit configuration with degree $L=90$ and order $M=45$

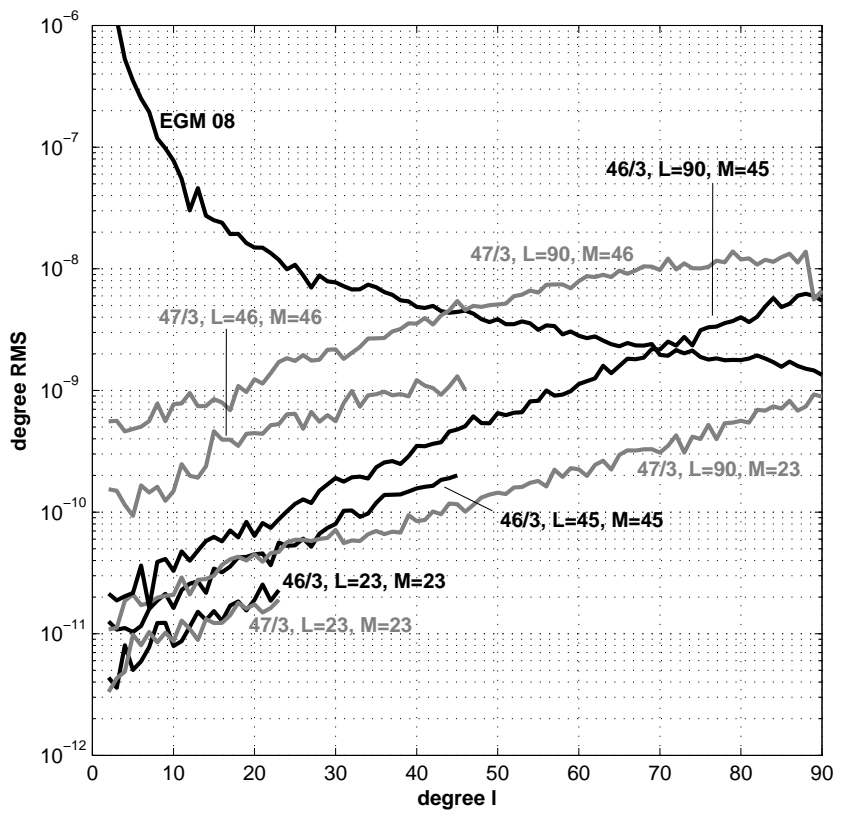

Fig. 7 Difference degree RMS w.r.t. the reference field EGM08 performs slightly poorer than the case of $L=M=45$ due to the poorer conditioning of the normal matrix but at the same time the even orbit configuration with degree $L=90$ and order $M=46$ performs again one to two orders of magnitude worse than the former. The general deterioration of the cases $L=90$ compared to the cases $L=45$ and $L=46$ reflects the influence of the parametrization on the conditioning of the normal matrix which should not be mixed with the influence of the orbit configuration. In what follows, equation (18) is indeed the correct criterion and the deterioration of two to three orders of magnitude at $M=\beta / 2$ cannot be neglected in the case of even parity.

\section{Implications for CHAMP and GRACE}

With this new understanding of sampling and aliasing, we are able to provide a table of maximum resolvable order for the satellite mission CHAMP and GRACE. Similar investigations have already been done by Klokočník et al. (2008) but here we consider a longer period of data, include the new understanding of the restriction on the maximum order $M$ in dependence of the parity and also show upcoming and potentially harmful repeat cycles for GRACE. For GOCE, this is of minor interest since the satellite is actively kept on its orbit height whereas the satellites of the other two missions are slowly decaying and passing several repeat periods over time. Besides, the primary influence due to the orbit on the quality of the GOCE-solution comes from the polar gap problem since the inclination of the orbit is $96.3^{\circ}$.

\subsection{GRACE}

For the calculation of the repeat cycles passed by GRACE an inverted version of equation (19) is used. The semi-major axis $a$ and $\dot{\Lambda}$ are directly derived from the satellites position and velocity provided in the Level-1B GPS navigation data. The data spans the time from August 2002 till August 2011 and was downloaded from the Information System and Data Center (ISDC) at the GeoForschungsZentrum (GFZ) Potsdam.

Fig. 8 shows the orbit decay and the repeat cycles passed so far. The later are also listed in table 2 together with the number of orbit revolutions $\beta$, the number of nodal days $\alpha$, the month and year in which the repeat cycle (numerically) occurs, the parity, the approximate orbit height, the number of unique equator crossings $\chi$ and the maximum resolvable order according to equation (18). Only repeat cycles with $\alpha \leq 16$ are considered because it can be estimated to be the maximum number of nodal days causing repeat ratios which have a negative impact on the quality of the gravity field solution. This is based on the experience that the signal-to-noise ratio limits GRACE monthly solutions to 


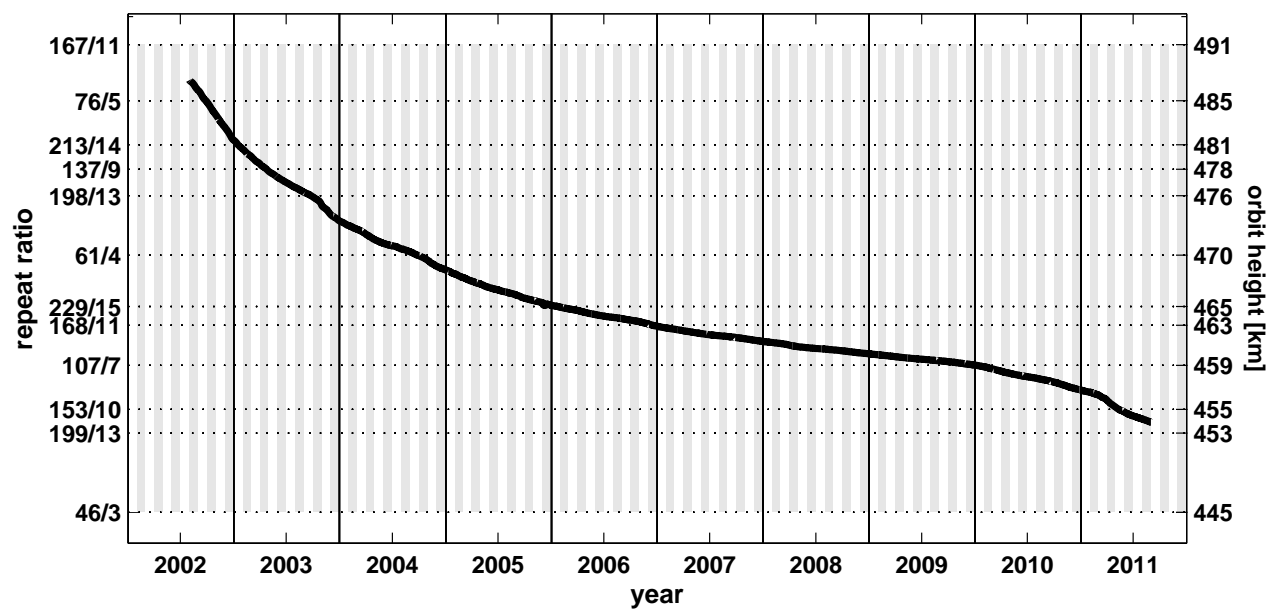

Fig. 8 Repeat ratios for GRACE from August 2002 till August 2010

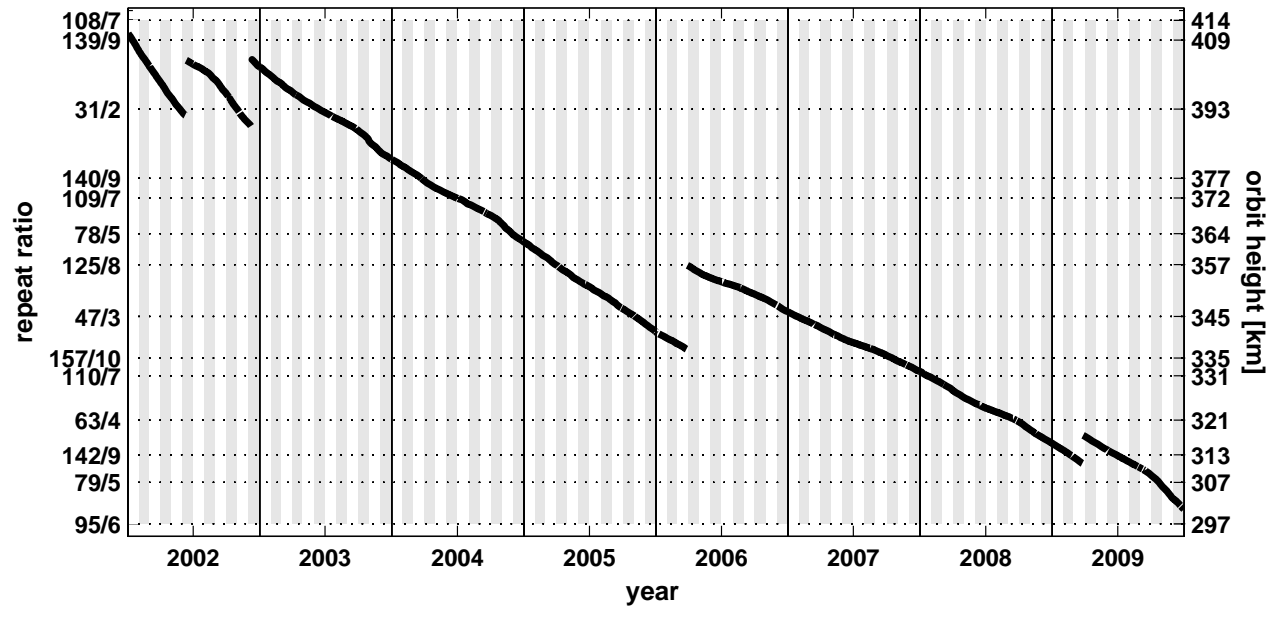

Fig. 9 Repeat ratios for CHAMP from January 2002 till December 2009

approximately degree and order 120. By assuming an even parity repeat cycle as a worst case scenario and inversely applying equation (18b), $\beta=240$ revolutions will be necessary. With the conservative assumption of more than $15 \mathrm{rev}-$ olutions per day, $\alpha=16$ follows. Note also that the stated period of occurrence are only those where repeat cycles numerically occur. Degradations are also possible in the vicinity of particular repeat cycles. Those cycles which - from a theoretical point of view - pose no problem for the gravity field recovery are grayed.

The first problematic repeat cycle $76 / 5$ is experienced in September 2002, followed by the 137/68 repeat cycle in April 2003. As stated in the introduction the $61 / 4$ repeat cycle attracted the attention of the GRACE community, which was passed near September 2004. In reaction to the sparse data distribution, the GFZ provides for the period from July till October 2004, additionally to the standard unconstrained solutions till degree and order 120 , constrained solutions till degree and order 60.
The severest condition, so far, was experienced from the end of 2009 till July 2010 as the satellites passed the 107/7 repeat cycle. Since $(\beta-\alpha)$ is of even parity, the maximum resolvable order is $M=53$. It results in an even sparser ground track than in the 61/4 repeat cycle which is of odd parity. Again, the GFZ provided constrained solutions till degree and order 60 for the period from October 2008 till July 2010. Furthermore, the GFZ suggests to use the unconstraint monthly fields also only up to degree 60 (Flechtner et al., 2010). According to table 2, similar problems are also expected for the periods around September 2002 and April 2003 but no constraint solution has been provided for these periods.

Table 2 lists additional repeat cycles down to an orbit height of $300 \mathrm{~km}$ which GRACE may pass if the satellites remain operational. Among the severest of the upcoming repeat cycles are the $31 / 2,46 / 3,47 / 3,63 / 4,77 / 5$ and $79 / 5$. During all these periods, constrained solutions will likely be necessary again. The time of occurrence is hard to predict and depends on the influence of the atmospheric drag, the so- 
lar activity and orbit maneuvers. Possible scenarios demand an ensemble approach since especially models for the solar activity are currently insufficient and instead a range of scenarios between an expected maximum and minimum so-

Table 2 Month of occurring repeat cycles for GRACE and their maximum resolvable order according to equation (18); gray repeat cycles do not pose a problem for the monthly gravity field recovery since $M>120$

\begin{tabular}{|c|c|c|c|c|c|c|}
\hline$\beta$ & $\alpha$ & Period & Parity & $\approx h[\mathrm{~km}]$ & $\chi$ & $M$ \\
\hline 76 & 5 & September 2002 & odd & 485 & 152 & 75 \\
\hline 213 & 14 & January 2003 & odd & 481 & 426 & 212 \\
\hline 137 & 9 & April 2003 & even & 478 & 137 & 68 \\
\hline 198 & 13 & September 2003 & odd & 476 & 396 & 197 \\
\hline 61 & 4 & September 2004 & odd & 470 & 122 & 60 \\
\hline 229 & 15 & January 2006 & even & 465 & 229 & 114 \\
\hline 168 & 11 & December 2006 & odd & 463 & 336 & 167 \\
\hline 107 & 7 & December 2009 & even & 459 & 107 & 53 \\
\hline 153 & 10 & May 2011 & odd & 455 & 306 & 152 \\
\hline 199 & 13 & - & even & 453 & 199 & 99 \\
\hline 245 & 16 & - & odd & 451 & 490 & 244 \\
\hline 46 & 3 & - & odd & 445 & 92 & 45 \\
\hline 215 & 14 & - & odd & 438 & 430 & 214 \\
\hline 169 & 11 & - & even & 436 & 169 & 84 \\
\hline 123 & 8 & - & odd & 433 & 246 & 122 \\
\hline 200 & 13 & - & odd & 430 & 400 & 199 \\
\hline 77 & 5 & - & even & 426 & 77 & 38 \\
\hline 185 & 12 & - & odd & 421 & 370 & 184 \\
\hline 108 & 7 & - & odd & 417 & 216 & 107 \\
\hline 247 & 16 & - & odd & 415 & 494 & 246 \\
\hline 139 & 9 & - & even & 412 & 139 & 69 \\
\hline 170 & 11 & - & odd & 410 & 340 & 169 \\
\hline 201 & 13 & - & even & 407 & 201 & 100 \\
\hline 232 & 15 & - & odd & 406 & 464 & 231 \\
\hline 31 & 2 & - & odd & 396 & 62 & 30 \\
\hline 233 & 15 & - & even & 387 & 233 & 116 \\
\hline 202 & 13 & - & odd & 385 & 404 & 201 \\
\hline 171 & 11 & - & even & 383 & 171 & 85 \\
\hline 140 & 9 & - & odd & 380 & 280 & 139 \\
\hline 249 & 16 & - & odd & 378 & 498 & 248 \\
\hline 109 & 7 & - & even & 376 & 109 & 54 \\
\hline 187 & 12 & - & odd & 372 & 374 & 186 \\
\hline 78 & 5 & - & odd & 367 & 156 & 77 \\
\hline 203 & 13 & - & even & 363 & 203 & 101 \\
\hline 125 & 8 & - & odd & 360 & 250 & 124 \\
\hline 172 & 11 & - & odd & 357 & 344 & 171 \\
\hline 219 & 14 & - & odd & 355 & 438 & 218 \\
\hline 47 & 3 & - & even & 348 & 47 & 23 \\
\hline 251 & 16 & - & odd & 342 & 502 & 250 \\
\hline 204 & 13 & - & odd & 341 & 408 & 203 \\
\hline 157 & 10 & - & odd & 339 & 314 & 156 \\
\hline 110 & 7 & - & odd & 335 & 220 & 109 \\
\hline 173 & 11 & - & even & 331 & 173 & 86 \\
\hline 236 & 15 & - & odd & 329 & 472 & 235 \\
\hline 63 & 4 & - & odd & 324 & 126 & 62 \\
\hline 205 & 13 & - & even & 319 & 205 & 102 \\
\hline 142 & 9 & - & odd & 317 & 284 & 141 \\
\hline 221 & 14 & - & odd & 314 & 442 & 220 \\
\hline 79 & 5 & - & even & 310 & 79 & 39 \\
\hline 253 & 16 & - & odd & 307 & 506 & 252 \\
\hline 174 & 11 & - & odd & 305 & 174 & 173 \\
\hline 95 & 6 & - & odd & 301 & 190 & 94 \\
\hline
\end{tabular}

lar activity has to be considered. This is beyond the scope of this paper and the interested reader is instead referred to Klokočník et al. (2008) where such an attempt has been made.

\subsection{CHAMP}

Similarly to GRACE, also the repeat cycles of CHAMP can be derived. Orbit data from January 2002 till December 2009 is kindly provided by Adrian Jäggi of the Astronomical Institute of the University of Bern and is essentially the same used in the calculation of the CHAMP-only gravity field model AIUB-CHAMP03s (Prange, 2010). The orbit decay and the repeat cycles are shown in Fig. 9 and listed in table 3. In this case, only repeat ratios with $\alpha \leq 10$ are considered as the signal to noise ratio limits monthly CHAMP solutions to approximately degree and order 70 . The maximum value for $\alpha$ can similarly be derived as for GRACE.

During the period of the available data the satellite passes 16 repeat cycles; some of them repeatedly as the orbit was lifted in between. In total, 10 of the repeat cycles are potentially harmful for the gravity field recovery. In May and October 2002 and in June 2003 the satellite passed the 31/2 repeat cycle three times. According to equation (18a) the order is limited to $M=30$, which is in good agreement with the findings of Weigelt et al. (2009). There, the intersection between the signal and the difference RMS curve w.r.t. to GGM02s model was found to be near degree $L=35$. The severest condition was passed in November 2005 and in January 2007 as the satellite passed the $47 / 3$ repeat cycle twice. Since this is a cycle with even parity, the ground track is

Table 3 Month of occurring repeat cycles with $\alpha \leq 10$ for CHAMP and their maximum resolvable order according to equation (18); gray repeat cycles do not pose a problem for the monthly gravity field recovery since $M>70$

\begin{tabular}{rrrcccr}
\hline$\beta$ & $\alpha$ & Period & Parity & $\approx h[\mathrm{~km}]$ & $\chi$ & $M$ \\
\hline 139 & 9 & January 2002 & even & 409 & 139 & 69 \\
31 & 2 & May 2002 & odd & 393 & 62 & 30 \\
31 & 2 & October 2002 & odd & 393 & 62 & 30 \\
31 & 2 & June 2003 & odd & 393 & 62 & 30 \\
140 & 9 & March 2004 & odd & 377 & 280 & 139 \\
109 & 7 & July 2004 & even & 372 & 109 & 54 \\
78 & 5 & November 2004 & odd & 364 & 156 & 77 \\
125 & 8 & April 2005 & odd & 357 & 250 & 124 \\
47 & 3 & November 2005 & even & 345 & 47 & 23 \\
125 & 8 & March 2006 & odd & 357 & 250 & 124 \\
47 & 3 & January 2007 & even & 345 & 47 & 23 \\
157 & 10 & October 2007 & odd & 335 & 314 & 156 \\
110 & 7 & January 2008 & odd & 331 & 220 & 109 \\
63 & 4 & September 2008 & odd & 321 & 126 & 62 \\
142 & 9 & February 2009 & odd & 313 & 284 & 141 \\
142 & 9 & June 2009 & odd & 313 & 284 & 141 \\
79 & 5 & October 2009 & even & 307 & 79 & 39 \\
\hline
\end{tabular}


sparsest during the considered period and solutions are only possible till order $M=23$. Compare also the repeat cycles $78 / 5$ and $79 / 5$ as these are similar to the test configurations used in section 3 .

\section{Conclusions}

A refined version of the Nyquist rule-of-thumb for the influence of the spatial sampling on the quality of a gravity field solution is provided by equation (18) which we have investigated by mapping the geopotential along an idealized model of a repeat trajectory. It describes solely the influence of the orbit configuration and is restricted to near polar orbits as it separately considers the spatial sampling in North-South and East-West direction. This separation is only possible for orbits with an inclination near $90^{\circ}$. For GOCE, the rule is already limited in its use as the polar gap problem becomes dominant. For GRACE and CHAMP though, the assumption is valid. Practically, the North-South sampling is given by the sampling of the measurement system which is in almost any case sufficient for nowadays gravity field recovery applications. The East-West sampling on the other hand is defined by the number of unique equator crossings and limits the spatial resolution by restricting the maximum resolvable order $M$ instead of the usually assumed maximum resolvable degree $L$. A dependency on the parity $\beta-\alpha$ is also recognized which plays an important role in the presence of noise. For $\beta-\alpha=$ odd, ascending and descending arcs are shifted by $\pi / \beta$ with respect to each other, i.e. ascending arcs are located inbetween descending arcs. For $\beta-\alpha=$ even, ascending and descending arcs cross the equator at the same longitude. The sampling in East-West direction is essentially cut in half. The concept of unique equator crossings enables the description of this.

The geometry of the measurement system is reflected in the conditioning of the normal matrix which is the ratio between the largest and smallest eigenvalue. The reduced sampling in case of even parity causes a degradation of the conditioning and leads to a reduced quality of the resulting gravity field solution in the presence of noise. The influence of the orbit configuration is then correctly described by equation (18). It is very important to realize that it only and solely describes the influence of the spatial sampling. Other factors also influence the conditioning of the normal matrix. Among them are mismodelling due to an over- or underparametrization, systematic errors, and the polar gap problem. The interplay of all these effects make the description of single influencing sources in practical applications very difficult. Only in extreme cases one or the other effect becomes dominant and can be identified as the major malefactor. For CHAMP and GRACE, this is the case for the low $\alpha$ repeat cycles, e.g. the $31 / 2$ for CHAMP or the $107 / 7$ in case of GRACE. The interaction of the different error sources is not fully understood yet and needs further investigations in the future. Thus, this paper can only be seen as one but important step towards a deeper understanding of the satellite systems. Nevertheless and with this new understanding of the spatial sampling, it is possible to identify or rule out impacts of the orbit configuration on the quality of the gravity field solution based on the orbital elements and improve the orbit design in the future. Furthermore, it can be concluded that the rule-of-thumb is too conservative and solutions to degrees higher than $\beta / 2$ are achievable for the case of odd parity. Visser et al. (2012) showed that this might also result in a non-homogeneous error distribution in the spatial domain which is acceptable as long as the quality of the solution is not degrading. This paper considered only one-dimensional observations. Visser et al. (2012) indicated already that different type of rules might be applicable for other, higherdimensional quantities. The impact on the conditioning of the normal matrix in the presence of noise needs to be investigated in these cases in the future.

\section{A Equator crossings}

This appendix gives the general description for the dependency on the parity. In section 2.1, the relation between one descending and all ascending arcs was used. This general description relates all descending to all ascending arcs. Consider again a circular orbit and an arbitrarily located equator crossing of an ascending arc at longitude $\lambda_{0}^{\mathrm{a}}$. The equator crossing of the $p$-th ascending arc can then be found by:

$\lambda_{p}^{\mathrm{a}}=\lambda_{0}^{\mathrm{a}}+\frac{2 \pi}{\beta} p$, with $p=0 \ldots \beta-1$.

Starting from $\lambda_{0}^{\mathrm{a}}$, the location of the first (in the timely sense) equator crossing of a descending arc is shifted by 180 degrees minus the angle passed by the Earth during the traveling of the satellite from equator crossing of the ascending to one of the descending arc. The equator crossings of the $q$-th descending arc is then given by:

$\Lambda_{q}^{\mathrm{d}}=\Lambda_{0}^{\mathrm{a}}+\pi-\pi \frac{\alpha}{\beta}+\frac{2 \pi}{\beta} q$, with $q=0 \ldots \beta-1$.

Similar to $p, q$ refers also to the East-West numbering of the equator crossing of the descending arcs and not to their timely occurrence.

The equator crossings of ascending and descending arcs will coincide if a set of $(p, q)$ can be found for which the difference of ascending and descending arcs becomes zero:

$\Lambda_{p}^{\mathrm{a}}-\Lambda_{q}^{\mathrm{d}}=0$.

Inserting equation (20) and (21) yields

$$
\begin{aligned}
& \Lambda_{0}^{\mathrm{a}}+\frac{2 \pi}{\beta} p-\Lambda_{0}^{\mathrm{a}}-\pi+\pi \frac{\alpha}{\beta}-\frac{2 \pi}{\beta} q=0 \\
& \Leftrightarrow \quad \frac{2 p}{\beta}-1+\frac{\alpha}{\beta}-\frac{2 q}{\beta} \quad=0 \\
& \Leftrightarrow \quad 2 p-\beta+\alpha-2 q \quad=0 \\
& \Leftrightarrow \quad|2 p-2 q| \quad=\beta-\alpha
\end{aligned}
$$

The absolute bracket is added because the difference $(\beta-\alpha)$ will always be positive. Since all quantities are integer values, equation (23) has only a solution in case of even parity of $(\beta-\alpha)$. 


\section{B Condition number}

Table $4 \log _{10}$ of the condition number for two repeat conditions each with and without order-limiting and different maximum degree for the case of noise-free data. Condition numbers beyond the computational accuracy are marked gray.

\begin{tabular}{r|rrr|rr}
\hline & \multicolumn{3}{|c|}{$\mathbf{4 7 / 3}$} & \multicolumn{2}{|c}{$\mathbf{4 6 / 3}$} \\
$L$ & $M=L$ & $M=46$ & $M=23$ & $M=L$ & $M=45$ \\
\hline 5 & 0.82 & 0.82 & 0.82 & 0.84 & 0.84 \\
10 & 1.18 & 1.18 & 1.18 & 1.25 & 1.25 \\
22 & 1.81 & 1.81 & 1.81 & 2.02 & 2.02 \\
23 & 1.86 & 1.86 & 1.86 & 2.08 & 2.08 \\
24 & 4.47 & 4.47 & 1.90 & 2.15 & 2.15 \\
25 & 4.51 & 4.51 & 1.94 & 2.22 & 2.22 \\
26 & 4.74 & 4.74 & 1.98 & 2.28 & 2.28 \\
30 & 5.08 & 5.08 & 2.16 & 2.51 & 2.51 \\
40 & 5.60 & 5.60 & 2.61 & 3.12 & 3.12 \\
44 & 5.78 & 5.78 & 2.80 & 3.37 & 3.37 \\
45 & 5.83 & 5.83 & 2.85 & 3.43 & 3.43 \\
46 & 5.86 & 5.86 & 2.90 & 19.74 & 3.49 \\
47 & 17.33 & 5.92 & 2.95 & 19.91 & 3.57 \\
48 & 18.52 & 5.95 & 2.99 & 19.82 & 3.66 \\
50 & 19.55 & 6.05 & 3.09 & 20.30 & 3.85 \\
60 & 20.17 & 6.57 & 3.57 & 21.66 & 4.87 \\
70 & 21.00 & 6.98 & 4.05 & 22.57 & 5.38 \\
80 & 22.56 & 7.39 & 4.53 & 22.86 & 6.02 \\
90 & 22.99 & 7.86 & 5.02 & 23.59 & 6.66 \\
95 & 23.61 & 8.91 & 6.43 & 23.89 & 6.99 \\
100 & 23.31 & 16.55 & 9.91 & 24.50 & 12.44
\end{tabular}

Acknowledgements We like to thank Dr. Adrian Jäggi for providing seven years of CHAMP data. We would also like to thank the German Space Operations Center (GSOC) of the German Aerospace Center (DLR) for providing continuously and nearly $100 \%$ of the raw telemetry data of the twin GRACE satellites. We also like to thank J. Kusche and J. Klokočnik as well as two anonymous reviewers for their helpful comments and suggestions.

\section{References}

Albertella, A., F. Sansò, and N. Sneeuw, Band-limited functions on a bounded spherical domain: the Slepian problem on the sphere, J. Geod., 73(9), 436-447, doi: 10.1007/PL00003999, 1999.

Bender, P., D. Wiese, and R. Nerem, A Possible DualGRACE Mission with 90 Degree and 63 Degree Inclination Orbits, in 3rd International Symposium on Formation Flying, Missions and Technologies, edited by ESA, ESA/ESTEC, Noordwijk, The Netherlands, 2008.

Bezděk, A., J. Klokočník, J. Kostelecký, R. Floberghagen, and C. Gruber, Simulation of free fall and resonances in the GOCE mission, J.Geodyn., 48(1), 47-53, doi:10.1016/j.jog.2009.01.007, 2009.
Bezděk, A., J. Klokočník, J. Kostelecký, R. Floberghagen, and J. Sebera, Some Aspects of the orbit selection for the measurement phases of GOCE, in Proc. ESA Living Planet Symposium, Bergen, Norway, 2010.

Colombo, O., The global mapping of gravity with two satellites, no. 7 (3) in Publications on Geodesy, 263 pp., Netherlands Geodetic Commission, http://www.ncg.knaw.nl/eng/publications/geodesy.html, 1984.

Flechtner, F., Ch. Dahle, K. Neumayer, R. König, and Ch. Förste, The Release 04 CHAMP and GRACE EIGEN Gravity Field Models, in System Earth via GeodeticGeophysical Space Techniques, edited by F. Flechtner, Th. Gruber, A. Güntner, M. Mandea, M. Rothacher, T. Schöne, and J. Wickert, doi:10.1007/978-3-642-10228-8_4, 2010.

Han, S., C. Jekeli, and C. Shum, Time-variable aliasing effects of ocean tides, atmosphere, and continental water mass on monthly mean GRACE gravity field, J. Geophys. Res., 109, B04,403, doi:10.1029/2003JB002501, 2004.

Heiskanen, W., and H. Moritz, Physical geodesy, W.H. Freeman and Company San Francisco, 1967.

Ilk, K., J. Flury, R. Rummel, P. Schwintzer, W. Bosch, C. Haas, J. Schröter, D. Stammer, W. Zahel, H. Miller, R. Dietrich, P. Huybrechts, H. Schmeling, D. Wolf, J. Riegger, A. Bardossy, and A. Güntner, Mass transport and mass distribution in the Earth system, 2nd ed., GeoForschungsZentrum Potsdam, Proposal for the German Priority Research Program, 2005.

Jekeli, C., The determination of gravitational potential differences from satellite-to-satellite tracking, Celestial Mechanics and Dynamical Astronomy, 75, 85-101, doi: 10.1023/A:1008313405488, 1999.

Johannessen, J., and M. Aguirre-Martinez, Gravity Field and Steady-State Ocean Circulation Mission, Tech. Rep. SP1233(1), ESA - European Space Agency, 1999.

Kim, M., Theory of satellite ground-track crossover, $J$. Geod., 71(12), 749-767, doi:10.1007/s001900050141, 1997.

Klokočník, J., J. Kostelecký, and H. Li, Best lumped coefficients from resonances for direct use in earth gravity field modelling, Bull. Astron. Inst. Czechosl., 41(1), 1726, 1990.

Klokočník, J., C. Wagner, J. Kostelecký, A. Bezděk, P. Novák, and D. McAdoo, Variations in the accuracy of gravity recovery due to ground track variability: GRACE, CHAMP, and GOCE, J. Geod., 82(12), 917-927, doi: 10.1007/s00190-008-0222-0, 2008.

Pavlis, N., S. Holmes, S. Kenyon, and J. Factor, An Earth Gravitational Model to Degree 2160: EGM2008, in presented at the 2008 General Assembly of the European Geosciences Union, Vienna, Austria, April 13-18, 2008.

Prange, L., Global Gravity Field Determination Using the 
GPS Measurements made Onboard the Low Earth Orbiting Satellite CHAMP, Ph.D. thesis, University of Berne, 2010.

Schrama, E. J. O., The role of orbit errors in processing of satellite alitmeter data, Tech. Rep. 33, Netherlands Geodetic Commission, 1989.

Schrama, E. J. O., Gravity field error analysis: application of GPS receivers and gradiometers on low orbiting platforms, Technical Memorandum 100769, NASA: Goddard Space Flight Center, 1990.

Schrama, E. J. O., Gravity field error analysis: applications of global positioning system receivers and gradiometers on low orbiting platforms, J. Geophys. Res, 96(B12), 20,04120,051, doi:10.1029/91JB01972, 1991.

Sneeuw, N., A semi-analytical approach to gravity field analysis from satellite observations, Reihe C 527, DGK, 2000.

Sneeuw, N., and M. van Gelderen, The polar gap, in Geodetic boundary value problems in view of the one centimeter geoid, Lecture Notes in Earth Sciences, vol. 65, edited by F. Sansò and R. Rummel, pp. 559-568, Springer-Verlag, Berlin, doi:10.1007/BFb0011717, 1997.

Sneeuw, N., Ch. Gerlach, D. Švehla, and Ch. Gruber, A first attempt at time-variable gravity field recovery from CHAMP using the energy balance approach, in Gravity and Geoid: Proceedings of 3rd Meeting of the International Gravity and Geoid Commission, Thessaloniki, 2002, edited by I. Tziavos, pp. 237-242, 2003.

Sneeuw, N., C. Gerlach, L. Földváry, T. Gruber, T. Peters, R. Rummel, and D. Švehla, One year of time-variable CHAMP-only gravity field models using kinematic orbits, in A window on the future of geodesy, International Association of Geodesy Symposia, vol. 128, edited by F. Sansò, pp. 288-293, Springer, doi:10.1007/3-54027432-4_49, 2005.

Tapley, B., S.Bettadpur, J. Ries, P. Thompson, and M. Watkins, GRACE measurements of mass variability in the Earth system, Science, 305, 503-505, doi: 10.1126/science.1099192, 2004.

Visser, P. N. A. M., N. Sneeuw, T. Reubelt, M. Losch, and T. van Dam, Space-borne gravimetric satellite constellations and ocean tides: aliasing effects, Geophys. J. Int., 181(2), 789-805, doi:10.1111/j.1365246X.2010.04557.x, 2010.

Visser, P. N. A. M., E. J. O. Schrama, N. Sneeuw, and M. Weigelt, Dependency of resolvable gravitational spatial resolution on space-borne observation techniques, in Geodesy for Planet Earth, Proceedings of the 2009 IAG Symposium, Buenos Aires, Argentina, 31 August 4 September 2009, International Association of Geodesy Symposia, vol. 136, edited by S. Kenyon, M. Pacino, and U. Marti, pp. 373-379, Springer, doi:10.1007/978-3-642-
20338-1_45, 2012.

Wagner, C. A., and S. M. Klosko, Gravitational harmonics from shallow resonant orbits, Celest. Mech. Dyn. Astr., 16(2), 143-163, doi:10.1007/BF01228597, 1977.

Wagner, C. A., D. McAdoo, Klokočník J., and J. Kostelecký, Degradation of geopotential recovery from short repeat-cycle orbits: application to GRACE monthly fields, J. Geod., 80(2), 94-103, doi:10.1007/s00190-006-0036-x, 2006.

Weigelt, M., M. G. Sideris, and N. Sneeuw, On the influence of the ground track on the gravity field recovery from high-low satellite-to-satellite tracking missions: CHAMP monthly gravity field recovery using the energy balance approach revisited, J. Geod., 83(12), 1131-1143, doi:10.1007/s00190-009-0330-5, 2009.

Yamamoto, K., T. Otsubo, T. Kubo-oka, and Y. Fukuda, A simulation study of effects of GRACE orbit decay on the gravity field recovery, Earth Planets Space, 57(4), 291295, 2005. 